\title{
The evolving relationship of wound healing and tumor stroma
}

\author{
Deshka S. Foster, ${ }^{1,2}$ R. Ellen Jones, ${ }^{1}$ Ryan C. Ransom, ${ }^{1}$ Michael T. Longaker, ${ }^{1,2}$ and Jeffrey A. Norton ${ }^{1,2}$ \\ ${ }^{1}$ Hagey Laboratory for Pediatric Regenerative Medicine, Department of Surgery, Division of Plastic and Reconstructive \\ Surgery, and ${ }^{2}$ Department of Surgery, Stanford University School of Medicine, Stanford, California, USA.
}

The stroma in solid tumors contains a variety of cellular phenotypes and signaling pathways associated with wound healing, leading to the concept that a tumor behaves as a wound that does not heal. Similarities between tumors and healing wounds include fibroblast recruitment and activation, extracellular matrix (ECM) component deposition, infiltration of immune cells, neovascularization, and cellular lineage plasticity. However, unlike a wound that heals, the edges of a tumor are constantly expanding. Cell migration occurs both inward and outward as the tumor proliferates and invades adjacent tissues, often disregarding organ boundaries. The focus of our review is cancer associated fibroblast (CAF) cellular heterogeneity and plasticity and the acellular matrix components that accompany these cells. We explore how similarities and differences between healing wounds and tumor stroma continue to evolve as research progresses, shedding light on possible therapeutic targets that can result in innovative stromalbased treatments for cancer.

Conflict of interest: The authors have declared that no conflict of interest exists.

Published: September 20, 2018

\section{Reference information:}

JCI Insight. 2018;3(18):e99911. https://doi.org/10.1172/jici.

insight.99911.

\section{Introduction}

Tumors are composed of transformed, cancer cells along with nontransformed stroma, a heterogeneous microenvironment composed of cancer associated fibroblasts (CAFs) (also known as tumor stromal fibroblasts), extracellular matrix (ECM) components, immune cells, blood vessels, and lymphatics. The stroma is critical for neoplastic proliferation. Moreover, we are beginning to appreciate the breadth of complex cellular interactions that occur in the tumor microenvironment (TME). The extent to which these findings differ in the wound healing process is beginning to be elucidated.

Malignant tumor progression relies on the TME, which promotes cancer cell growth by inducing leaky vasculature, cell migration, and angiogenesis. More than 30 years ago, Dvorak described the connection between the TME and wound healing, proposing a model in which tumor stroma forms through abnormal activation of wound healing pathways (1). Both the wound healing and TME rely on changes in ECM deposition that promote epithelial-mesenchymal transition (EMT) and increase motility of both fibroblasts and tumor cells. This dynamic environment is driven by plasticity in local cells that are activated in response to stress transiently during the wound healing process and indefinitely in cancer (2).

Each step of the intricate processes of wound healing and tumor development requires the interaction of fibroblasts and associated ECM components $(3,4)$. The first stage of wound healing is hemostasis, in which platelets arrest bleeding and form a clot at the site of injury, which transforms to fibrin. Fibrin, along with vitronectin and fibronectin, form early matrix, and fibroblasts are recruited to the wound. Subsequently, neutrophils are recruited and release cytokines (Figure 1A), inducing the inflammatory stage of wound healing. Fibroblasts are activated in the wound site and deposit ECM components such as collagen III. Macrophages are recruited and clear devitalized tissue and debris from the wound site. Keratinocytes migrate superficially to cover the wound, and new blood vessels extend to the wound bed (Figure 1B). Lastly, in the remodeling stage, fibroblasts facilitate wound contraction, collagen I replaces collagen III, and the ECM is remodeled with the help of proteases and other enzymes to form a mature scar. Some nerve regeneration occurs and new blood vessels are pruned (Figure 1C). In each of these phases, fibroblasts play a key role orchestrating the healing process.

In tumors, fibroblasts are also crucial for tumor progression, which can also be organized into analogous stages (4). When neoplasia begins, fibroblasts are recruited to the site. As the transformed cells multiply, the fibroblasts are activated and incorporated into the tumor. These CAFs produce dysregulated ECM proteins and proteases, forming a scaffold for the cancer cells to continue proliferation. Collagen stiffening and linear- 
A

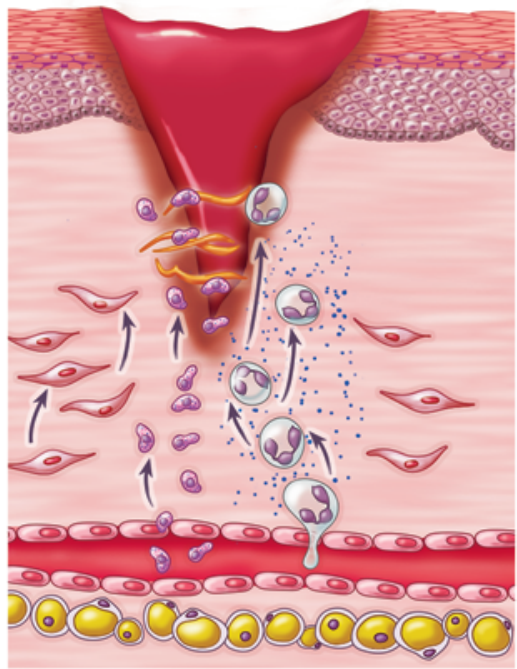

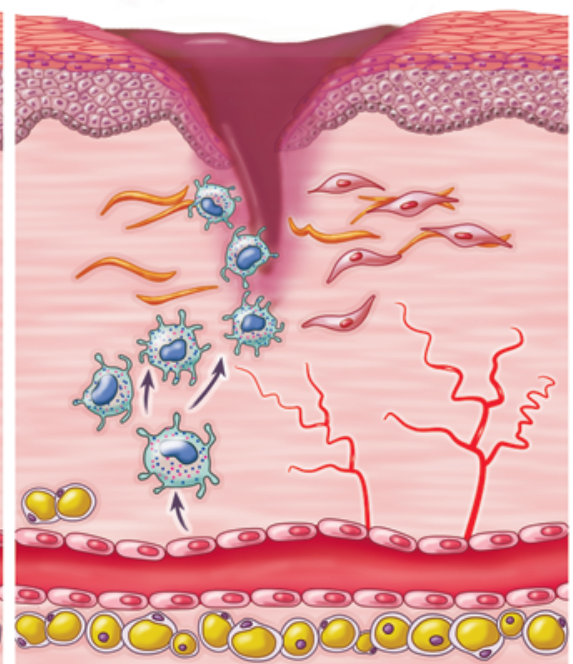

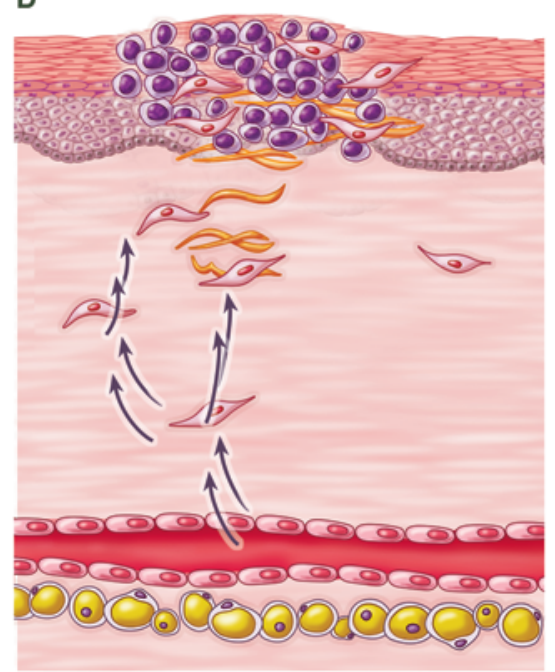

E

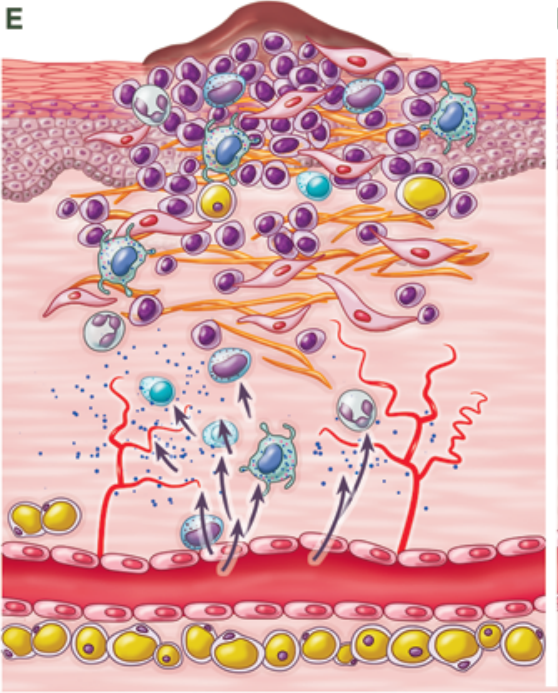

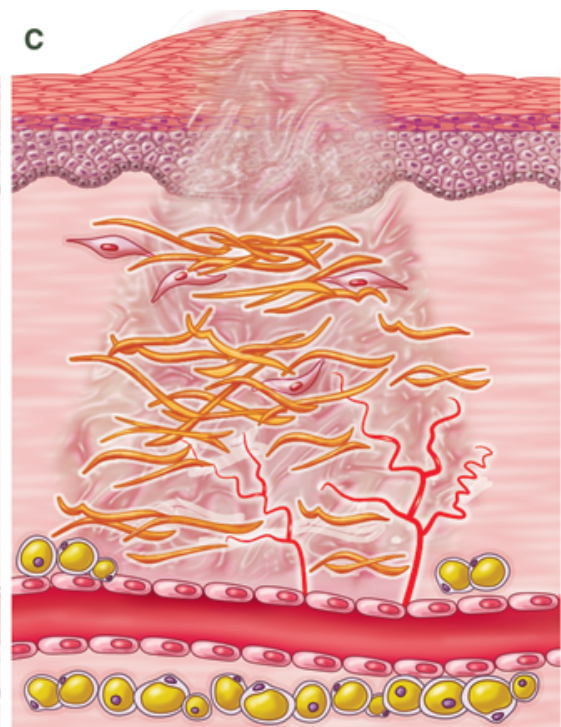

F

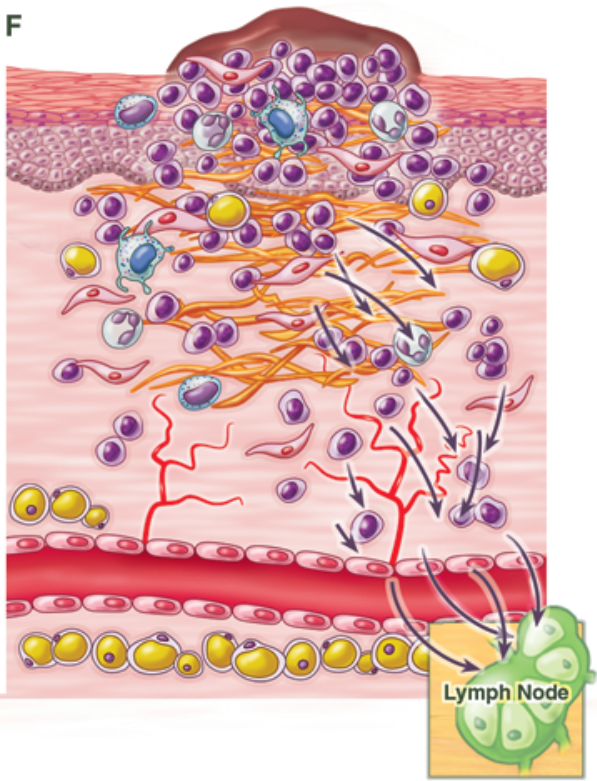

M. Migamoto

Fibroblasts

Figure 1. Comparative models of wound healing and tumor stroma. (A) The first phase of wound healing involves hemostasis; platelets form clot at the injury site, which transforms to fibrin. Fibrin, vitronectin, and fibronectin form early matrix. Neutrophils migrate to the wound and cytokines are released. Fibroblasts are recruited to the wound. (B) During the proliferation phase of wound healing, macrophages are recruited to clear dead tissue and debris. Keratinocytes migrate to cover the superficial wound area. New blood vessels form in the wound bed. Fibroblasts are activated in the wound and begin to deposit new ECM. (C) During the remodeling phase of wound healing, wound contraction occurs, collagen III is replaced by collagen I, and the ECM is remodeled by proteases and other enzymes. Nerve regeneration progresses and new vessels are pruned. (D) When neoplasia is first initiated, fibroblasts are recruited to the tumor site, activated, and incorporated. Production of dysregulated ECM proteins and proteases is stimulated, forming a scaffold for tumor proliferation. (E) As the tumor grows, the epithelium can be eroded away by cells expanding from below, causing ulceration at the skin edge of the tumor. VEGF and other signaling molecules induce neovascularization. Inflammatory cells are recruited to the TME and release cytokines. CAFs can adapt to tolerate and even participate in many of these features. Adipose cells also form a component of the tumor stroma. (F) The abnormal ECM is protumorigenic and proangiogenic. The tumor vasculature is leaky, which can allow metastases to distant sites. Tumor cells are also cleared by lymphatics and can permit lymphatic metastases. Illustrated by Mao Miyamoto. 
ization by lysyl oxidase (LOX) characterize this dysfunctional ECM, which is firmer than surrounding tissues similar to a scar. The abnormal ECM is both protumorigenic and proangiogenic. The new tumor vasculature is leaky, which can allow hematogenous metastases to distant sites. Inflammatory cells are recruited to the tumor stroma and release cytokines. Immune infiltration is regulated by the leaky nature of the new vessels. Desmoplasia, a local fibrotic reaction associated with solid tumors, results in increased connective tissue. Cancer cells cause the TME to have a low $\mathrm{pH}$ and hypoxia, secondary to the Warburg effect. CAFs can adapt to tolerate and even participate in many of these features. Cancer cells can be cleared by local lymphatics, which drain to lymph nodes, initiating lymph node metastases in many tumor types. Epithelial cancer cells may undergo EMT and invade through the basement membrane, allowing hematogenous dissemination (4) (Figure 1, D-F). Here, we focus on how CAFs in tumor proliferation relate to wound healing.

\section{Fibroblasts in solid tumors and cutaneous wounds}

Fibroblasts are a dominant cell type involved in both wound healing (Figure 1, A-C) and tumor stroma (Figures 1, D-F). Fibroblasts are critical for tissue repair after injury and are involved in wound contraction, deposition of granulation tissue, production of ECM components, and tissue remodeling. In tumors, fibroblasts influence the microenvironment through direct contact with cancer cells and paracrine signaling, regulate the immune response to neoplasia, deposit diverse ECM components, stimulate neoangiogenesis (5), and provide a scaffold for tumor progression. Fibroblasts are known to have both tumor-promoting and tumor-inhibiting roles (6). In wound healing, equivalent fibroblasts are also called activated myofibroblasts, which highlights the transition these cells undergo from a resting, quiescent state to an activated form after tissue injury. The "myo-" prefix emphasizes the expression of $\alpha$-smooth muscle actin ( $\alpha$-SMA), which is associated with these cells in both tumor and wounds (7-9).

Fibroblast activation. In homeostatic tissue, quiescent fibroblasts are spindle-shaped cells (6). Expression of fibroblast specific protein 1 (FSP-1) and $\alpha 1 \beta 1$ integrin are associated with quiescent fibroblasts in mice $(10,11)$. When a wound occurs or neoplasia is initiated, fibroblasts are recruited and activated in the microenvironment. Factors known to be involved in activating fibroblasts in both wound healing and tumor stroma include TGF- $\beta$ signaling (7), PDGF (12), FGF2 $(13,14)$, HGF, IGF, connective tissue growth factor (CTGF), Wnt signaling $(15,16)$, integrin expression $(17)$, and cell-cell interactions (Figure 2 ). In wound healing, TGF- $\beta 1$, via the SMAD-4 pathway, stimulates wound healing activities including wound contraction by fibroblasts and ECM component deposition, as well as immune cell recruitment, angiogenesis, and keratinocyte migration via integrins (18). TGF- $\beta 2$ shares similar roles to TGF- $\beta 1$ and is involved in stimulating ECM deposition, while TGF- $\beta 3$ is involved in regulating TGF- $\beta 1$ and decreasing collagen I deposition and scar formation (18).

In the tumor context, PDGF, through its primary effector osteopontin, is specifically involved in recruitment, activation, and phenotypic remodeling of CAFs (19). Local recruitment of fibroblasts by chemokines also contributes to fibroblast activation in both wound healing and tumor proliferation. An example of CAF activation occurs during the development of skin cancer: Melanoma cells secrete TGF- $\beta$, PDGF, FGF2, and IL-8, which act in a paracrine manner to stimulate fibroblast activation and proliferation (19). Specifically, when melanocytes are transformed, PDGF signaling (20) simulates Snail upregulation (21), which causes downregulation of melanoma cell E-cadherin expression, allowing them to interact with fibroblasts more directly. This interaction is mediated by N-cadherin and connexins (22). Epigenetic modification, specifically aberrant DNA methylation, also drives fibroblast activation $(2,23)$.

Once activated, fibroblasts acquire different morphology than their quiescent counterparts and shift to a migratory phenotype. This change promotes wound healing, but also facilitates tumor proliferation (24). There is a common CAF gene expression signature that aligns with the wound healing response, and is predictive of prognosis in human cancer (25). When normal wound healing is complete, activated fibroblasts are culled, either via apoptosis (26) or a transition back to a resting state. In tumors, fibroblast activation is never turned off.

Fibroblast phenotypic and functional heterogeneity. There is significant heterogeneity in fibroblast cell surface marker and protein expression in postinjury repair and neoplastic proliferation (6). For example, $\alpha$-SMA expression is primarily associated with activated fibroblasts (27) but is also expressed by tissue-resident fibroblasts (28), smooth muscle cells, and pericytes (29). Some cancer stromal fibroblasts express PDGFR $\alpha$ as well as or instead of $\alpha$-SMA (30). FSP1 is primarily associated with quiescent fibroblasts but is also expressed by CAFs and identifies a unique cohort of CAFs in mice, which is independent from cells expressing $\alpha$-SMA (31). Fibroblasts expressing the cell surface marker CD26 


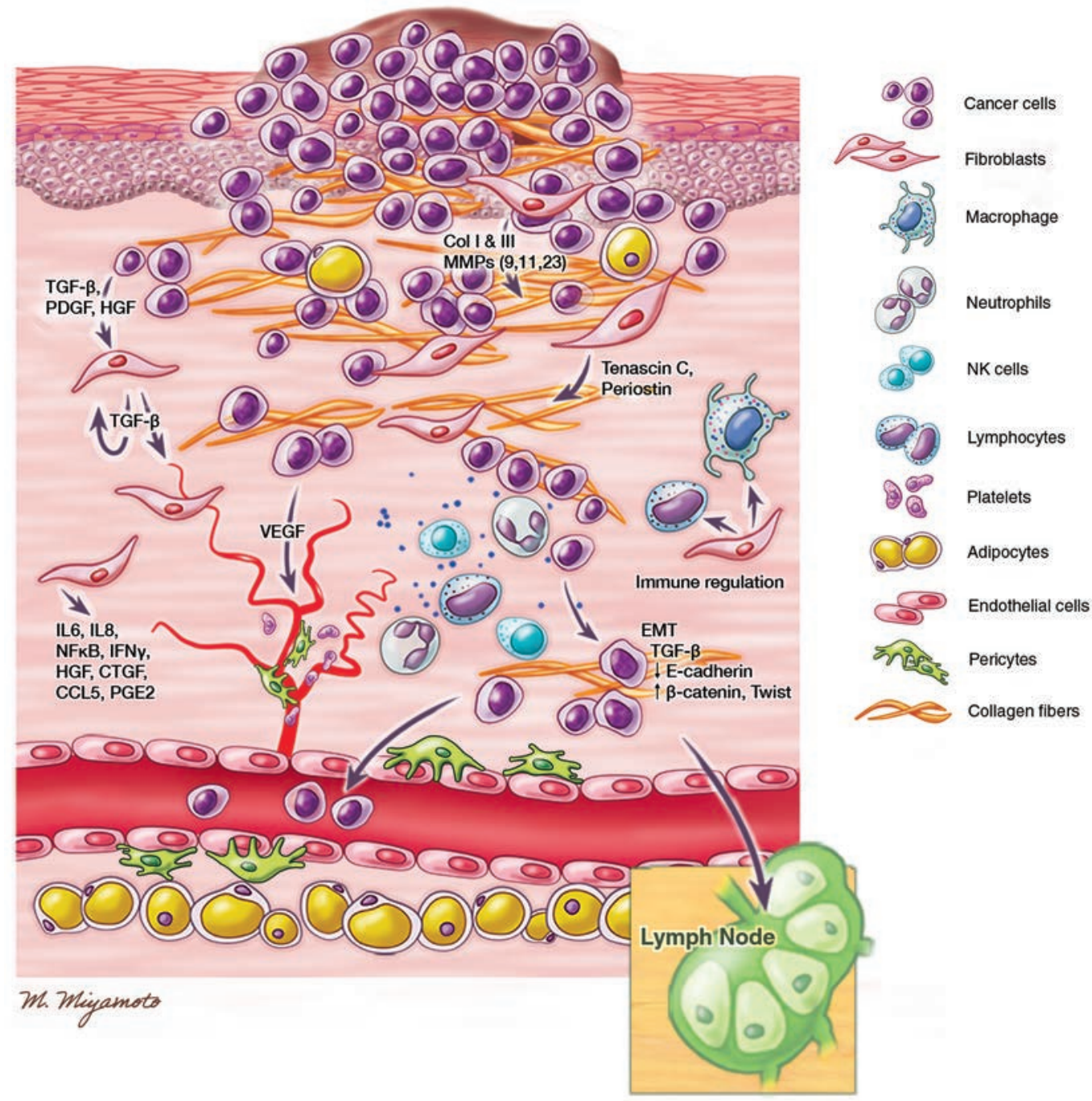

Figure 2. Detailed illustration of the tumor microenvironment showing representative cell types, tissues, and signaling factors involved. Fibroblasts are recruited by cancer cells and activated in the local environment via TGF- $\beta$, PDGF, HGF, and other signaling molecules. CAFs secrete TCF- $\beta$, which provides both a positive feedback mechanism encouraging fibroblast activation, as well as guiding the TME. CAFs also secrete paracrine signaling factors including IL-6, IL-8, NFKB, IFN- $\gamma$, HGF, CTCF, CCL5, and PGE2, as well as ECM molecules such as collagens, MMPs, tenascin C, and periostin. Immune cells respond to the tumor, including neutrophils, lymphocytes, macrophages, and NK cells. Cytokines are secreted. Both cancer cells, via VEGF secretion, and CAFs are involved in stimulating angiogenesis. These new vessels are leaky and dysfunctional, which can limit immune access to the tumor. CAFs regulate the immune response. Cancer cells, under the influence of CAF signaling, can undergo EMT with downregulation of E-cadherin expression and upregulation of $\beta$-catenin and Twist, and escape through the basement membrane with access to vasculature for metastatic spread. Cancer cells can also collect in the lymph nodes and metastasize through the lymphatics. Illustrated by Mao Miyamoto.

are engaged in scar and keloid formation and also implicated in tumor stroma $(32,33)$ (Table 1). This heterogeneity is corroborated in humans, with some homology between species (16). Such surface marker heterogeneity makes this cell population challenging to study - no single cell surface marker is sufficient to capture a specific or unique cell population. Better understanding this heterogeneity and its functional significance is an area of active investigative research.

In the context of wound healing, dorsal dermal scarring in mice is attributable to the activity of fibroblasts derived from a lineage defined by embryonic expression of Engrailed-1 (En1) (33). En1 fibroblasts also express FSP1, vimentin, Col1, and fibronectin. Notable exceptions in which scarless healing occurs include early gestational fetal skin (prior to E16.5) and the buccal mucosa $(34,35)$. Postnatally, En1 positive fibroblasts (EPFs) stop expressing En1 but are associated with expression of CD26/DPP4. Fibroblasts of the En1 lineage contribute not only to scarring, but also to tumor stroma in mouse melanoma (33). 
Table 1. Surface markers associated with activated fibroblasts in mice and humans

\section{Markers \\ $\alpha$-SMA ( $\alpha$-smooth muscle actin, also known as acta2) \\ FAP (fibroblast activation protein, also known as F19) \\ Significance in wound healing \\ An intracellular protein expressed by fibroblasts transiently in mouse wound healing. Associated with increased fibroblast contractility. Also expressed by normal fibroblasts, smooth muscle cells, and pericytes. Upregulated in human scar tissue. \\ Absent from adult tissue-resting fibroblasts but strongly induced during scar formation.} Upregulated in activated fibroblasts.

PDGFR $\alpha$ and $-\beta$ (PDGFR $\alpha$ also known as $C D 140 \alpha$ )

PDGFs induce fibroblast reactivity and fibrosis. Expression associated with fibroblasts involved in organ fibrosis and wound healing in mice and humans. Expressed by dorsal, scar-forming fibroblasts in mice. PDGFR $\beta$ is also expressed by normal pericytes.

FSP1 (fibroblast specific protein-1, FSP1 and $\alpha 1 \beta 1$ integrin expression are associated also known as S100A4)

with a quiescent fibroblast phenotype in mice.

$\mathrm{FSP}^{+}$fibroblasts are predominant cell types within experimental granulation tissue during wound healing at day 22 in a mouse model.

Vim (vimentin)

Col-I and Col-III (collagen I is protein product of Col1A1 and 1A2, formation and fibrosis. Secreted by En1-expressing collagen III is protein product of COL3A1 gene)

EGF

ED-A (fibronectin spiced variant)

A mesenchymal marker, upregulated in activated fibroblasts.

Postn (periostin)
Desmin
Podoplanin
FGF2
CD26 (also known as dipeptidyl
peptidase-4 [DPP4])
CD90 (also know as thymocyte
antigen 1 [Thy1])

A tissue repair product; levels in wounds correlate with activated fibroblast presence in granulation tissue.

Expressed by fibroblasts in hypertrophic stars and fibrosis in humans.

Displayed by human dermal fibroblasts in sclerosis (along with CD 90).

Involved in fibroblast activation in both acute and chronic tissue injury.

Associated with mouse papillary fibroblasts population. Expressed by En1 $1^{+}$fibroblast in the mouse dermis. Expressed by human dermal SFRP2+ fibroblasts.

Expressed by human dermal fibroblasts. Displayed by dermal fibroblasts in sclerosis (along with Podoplanin) in humans.

NG2 (neuron glial antigen-2) Expressed by migratory fibroblasts in response to injury in vitro.

Tenascin- $\mathrm{C}$
Involved in recruiting fibroblasts to wounds.

\section{Significance in tumor stroma}

Upregulated in reticular (in mice), activated fibroblasts in both the tumor stroma and wound healing contexts in mice and humans. High CAF $\alpha$-SMA expression is associated with poor prognosis in human breast cancer.

F19+ fibroblasts found in primary and metastatic colorectal, breast, bladder, ovarian, and lung carcinomas. Intratumoral FAP expression correlates with poor prognosis in human colon cancer. CAF FAP expression related to checkpoint inhibitor resistance in mice.

Involved in recruitment and phenotypic remodeling of CAFs. Found to be robustly expressed by CAFs in mouse squamous cell carcinoma.

FSP1 expression found to identify a unique cohort of CAFs independently from cells expressing $\alpha$-SMA

and PDCFR $\alpha / \mathrm{b}$. FSP1 ${ }^{+}$CAFs facilitate malignant progression. In mouse melanoma, PDGF-CC paracrine signaling recruits CAFs expressing PDCFR $\alpha, \alpha-S M A$, and FSP1. Also expressed by some carcinoma cells.

FSP1 deficiency in a mouse mammary carcinoma model yielded decreased tumor growth.

Expression necessary for fibroblast initiation of EMT in tumor cells. Also expressed by epithelial cells that have undergone EMT.

Upregulated in CAFs. Mesenchymal stem cells can gdifferentiate into fibroblastic cells expressing $\alpha$-SMA and Col-I in human lung tissue.

$39,43,144$ 15

$10,11,19,31$ $39,152,153$

$36,39,154$

$7-9,27,28$

149,150

$19,30,33,39$

$33,137,155$ 137 binding EGF overexpression in CAFs, fostering tumo progression.

Expressed by CAFs, as well as carcinoma cells and tumor blood vessels. Increased urinary ED-A associated with poor prognosis in human bladder cancer.

Expressed by CAFs in colon cancer. Upregulated in human pancreatic stellate (fibroblastic) cells; deposition seen at carcinoma cell infiltration sites. Also expressed by perivascular cells.

160

Marks a specific CAF population. Prognostically relevant in lung, breast, and SCC. Also expressed by lymphatic endothelial cells.

Binds tyrosine kinase receptor and induces VEGF expression.

Fibroblasts of En1 lineage (expressing CD26/DPP4) contribute to tumor stroma in mouse melanoma. When these fibroblasts are ablated (via diphtheria toxin), tumor burden decreases.

High CD90-expressing CAFs promote tumors more than low CD90-expressing CAFs in human gastric and prostate cancer.

Expressed on normal and intratumoral pericytes.

Pericytes distinguished by regulator of G-protein signaling 5 (RGS5) expression, which associates with CD31 rather than $\alpha$-SMA.

CAF expression involved in regulating carcinoma cell $\quad 37,170$ adhesion.
$161,162,163$

39, 164

$33,165,166$

161,167

168,169 
In humans, a major population of fibroblasts expressing SFRP2/DPP4 is associated with collagen bundles, likening this population to the scar-forming En1 population in mice (33). This population was not found to stratify specifically to the papillary or reticular regions in the skin. Other markers associated with activated fibroblasts in mice and humans include $\alpha$-SMA, vimentin (36), PDGFR $\beta$, fibroblast-associated protein (FAP), neuron glial antigen-2 (N2G), tenacin-C (37), desmin, periostin (38), THY1 (CD90), and podoplanin $(24,31,39)$ (Table 1).

Striking lineage plasticity is observed in activated fibroblasts in the setting of both healing wounds and tumor proliferation (2). For example, in wound healing, myofibroblasts are capable of differentiating into adipose cells, through BMP signaling (40). Such lineage flexibility highlights the importance of considering cellular activities in a context-dependent fashion. In tumors, heterogeneity also exists between the number and proportion of CAFs between different cancer types $(6,41)$. For example, breast, pancreatic, and lung cancers have relatively high numbers of fibroblasts, while other tumors such as brain and ovarian contain few fibroblasts (24).

One reason driving the investigative interest in understanding and classifying CAF surface marker expression is that the patterns and relative levels of marker expression may be prognostic. For example, in a breast cancer study, higher FSP1 expression correlated with shorter overall survival (42). Similarly, a colon cancer study showed that high intratumoral FAP expression correlates with worse prognosis (43). However, complicating this finding, it is important to note that FAP expression is not limited to CAFs and has also been detected in epithelial cancer cells (24). These functional differences are key in determining the clinical significance of such observations.

$C A F s$. The precise origin of fibroblasts in solid tumors remains unclear. Some studies suggest a heterogeneous origin, in which BM cells are recruited to transdifferentiate into fibroblasts (44); however, other findings implicate a local expansion of tissue-resident cells. Regardless of their origin, CAFs play a fundamental role in tumorigenesis. When human mammary epithelial cells were transformed and implanted into nude mice, fibroblast coinjection yielded more efficient tumorigenesis (45). In a model of squamous skin carcinogenesis, ultrasound studies showed that coinoculation of tumor cells with CAFs yielded less necrotic and more vascularized tumors than when cancer cells were administered alone (30).

CAFs can direct specific cancer cell activities. For example, CAFs induce angiogenesis and tumor growth in association with expression of stromal cell-derived factor 1 (SFD1), also known as CXCL-12 (46). CAFs also express transcription factors such as SNAIL1, which drive EMT in cancer cells leading to invasion and metastasis (47). Just as CAF surface markers can differ based on cancer type, so too can their roles within a tumor. CAFs express a specific proinflammatory gene signature in pancreatic and breast cancer (30). Proinflammatory CAFs recruit macrophages (30), which in turn are proangiogenic in mouse skin and cervical cancer models secondary to MMP-9 secretion and VEGF stimulation (48).

Once activated in tumors, CAFs secrete a wide variety of cytokines that regulate other stroma cell activities. These include IL-6, involved in modulating inflammation, cancer progression, and tumor angiogenesis (24, 39), IL-8, IFN- $\gamma$, HGF, CTGF, CCL5, prostaglandin E2 (PGE2) (39) and NFאB, which promote inflammatory pathways that enhance tumorigenesis (30) (Figure 2 and Table 2). IL-6 is also fundamentally important in cutaneous wound healing (49).

Several papers have suggested that the tumor suppressor p53 can be mutated or epigenetically inactivated in CAFs (50-53). In vitro, coculture experiments using p53-deficient fibroblasts yielded greater cancer cell proliferation than coculture with WT fibroblasts, suggesting that stromal p53 expression exerts a detrimental effect on tumor growth (54). In vivo studies in p53-KO mice showed that $\alpha$-SMA and tetraspanin (12) expression was notably increased, which correlated with increased cancer cell proliferation (55). In breast cancer, stromal cell p53 mutations were associated with poor prognosis and a higher incidence of nodal metastasis (51). On the other hand, robust p53 expression in stromal cells has been shown to potentially suppress tumor growth by generating an anti-TME, which occurs via macrophage function regulation (56).

Paradoxical role of fibroblasts in tumor stroma. While CAFs are known to support tumor progression, they also have poorly understood tumor-inhibiting effects (57). Tumors such as pancreatic ductal adenocarcinoma (PDAC) are characterized by extensive tumor and peritumoral fibrosis, known as desmoplasia. While desmoplasia is believed to be one of the reasons that cytotoxic therapies have limited effect in this cancer, when $\alpha$-SMAexpressing activated fibroblasts were depleted in mice with PDAC, tumor growth increased and animal survival decreased (58). Similarly, in human PDAC, patients with fewer myofibroblasts in their tumors have decreased survival (58). This is thought to occur because depletion of fibroblasts may worsen tumor immunosuppression. Conversely, patients with robust desmoplasia have improved survival not only in PDAC, but also in lung and breast cancers (59). CAFs may induce tumor cell sensitization to therapy rather than resistance (60). 
Table 2. Cancer associated fibroblast cytokines, chemokines, and signaling molecules

Cytokines, chemokines, and signaling molecules

TGF- $\beta$

HGF (hepatocyte growth factor, also known as scatter factor [SF])

PDGF (composed of dimeric subunits, either PDGF-AA, -AB, -BB, $-C C$, or -DD)

VEGF (also known as vascular permeability factor, VPF)

IGF1 (encoded by the IGF1 gene in humans)

IL-6 (encoded by the IL-6 gene in humans)

IL-8 (encoded by the CXCL8 gene in humans)

NFאB (nuclear factor $\kappa$-light-chain-enhancer of activated B cells)

CXC10 (CXC motif chemokine 10, also known as IFN- $\gamma$-induced protein 10 [IP10], encoded by the CXCL10 gene in humans) SDF1 (stromal cell-derived factor-1, also known as CXC12, encoded by the CXCL12 gene in humans)

IFN- $\gamma$

CTGF (connective tissue growth factor, also known as Cystinerich, Connective Tissue, Nephroblastoma protein-2 [CCN2])

CCL2 (also known as monocyte chemoattractant protein-1 [MCP1] or small inducible cytokine $A 2$, encoded by the CCL2 gene in humans)

CCL7 (also known as monocyte chemoattractant protein 3 (MCP3))

PGE2 (Prostaglandin E2)
Secreted by senescent CAFs from pancreas tumors in vitro and in a xenograft mouse model. These IL-8-upregulated CAFs promote invasion and metastatic spread (180). Also expressed by human breast tumor adipose-derived stem cells (ASCs) (181).

Promotes inflammatory pathways that enhance tumorigenesis. In a mouse squamous cell carcinoma model, CAFs gene expression patterns promoted angiogenesis, recruitment of macrophages, and tumor proliferation in a manner dependent on NFKB signaling (30). tumors (62). CAF secretion of TGF- $\beta$ induces EMT in breast carcinoma cells in humans (171) and promotes colorectal cancer progression and metastasis and correlates with poor prognosis (172). CAFs derived from mesenchymal stem cells (MSC) are recruited to gastric tumors by TGF- $\beta$, as well as SDF1 $\alpha$ (173). When TGF- $\beta$ II receptor was knocked out in mouse fibroblasts, the animals developed prostate neoplasia and stomach squamous cell carcinoma, which both showed increased numbers of tumor stroma cells. This is thought to occur secondary to paracrine HGF signaling. As such, CAF TCF- $\beta$ signaling is both an activator and a modulator of tumorigenesis (63).

Fibroblast-derived HGF expression is associated with cancer initiation. HGF secreted by CAFs regulates tumor proliferation in various tumor types in mice and human cells (174). HGF secretion is associated with CAF-mediated tyrosine kinase (175) and epidermal growth factor receptor inhibitor resistance (176). PDGF-CC activates CAFs via paracrine signaling and drives tumor growth in mouse melanoma. The ECM protein osteopontin is a primary effector in this pathway (19).

Osteopontin, secreted by CAFs, stimulates endothelial cell VEGF-A signaling (19). In VEGF-A-KO mice, CAFs that secrete VEGF are recruited in a manner that is dependent on PDCFR $\alpha$ expression (177).

Fibroblast secretion of IGF1 targets the ICF1 receptor on keratinocytes and promotes a protective response to UVB exposure in human. In older skin, IGF1 secretion is attenuated, resulting in ineffective protection against UVB exposure and driving tumorigenesis (178).

Secreted by CAFs and involved in modulating tumor inflammation. CAF IL-6 secretion drives EMT and promotes metastatic spread via JAK2/STAT3 pathway signaling in gastric cancer (179). CAFs derived from MSCs express IL-6, as well as Wnt5a and BMP4, and promote tumor proliferation in a mouse model of inflammation-induced gastric cancer (173).

A prometastatic chemokine, expression is stimulated by HIF1 (Hypoxia inducible factor 1) (182)

Associated with CAF induction of angiogenesis and tumor growth. CXC chemokine expression also known to be involved in orchestrating functions in regulation of the wound healing process (46).

An immunomodulatory cytokine produced by CAFs (183).

Member of the CAF secretome, associated with cancer cell proliferation and invasion (39).

Prostate cancer CAF secretion of CCL2 (and CXCL12), stimulated by CXCL8 paracrine signaling, is associated with prostate cancer cell proliferation in vitro (184). Also associated with cell proliferation in in vitro breast cancer studies. CCL2 is also expressed by macrophages in the TME.

Secreted by CAFs, as well as cancer cells and macrophages. Known to enhance carcinoma cell EMT, tumor proliferation, and invasion in a variety of cancers including gastric cancer, and it's overexpression (along with CCL21) is associated with poor prognosis (185).

In a study involving CAFs derived from human hepatocellular carcinoma specimens, CAF secretion of PGE2 (along with IDO) suppressed NK cell activation, resulting in tumor progression (186). In vitro and mouse model research shows that CAF production of PGE2, induced by direct contact with B16 melanoma cells, plays an important role in melanoma cell proliferation (187).
Involved in fibroblast activation and deposition of abnormal ECM in wounds and 
In mouse and human breast cancer, paracrine signaling causes CAF upregulation of Chi3L1 in both primary and metastatic tumors, which promotes tumor growth via signaling with both cancer and immune cells (61). When fibroblast Chi3L1 expression was knocked down in an orthotopic mouse model, fewer activated ( $\alpha$-SMA-expressing) fibroblasts were detected and tumor growth decreased with increased mouse survival (61), a seemingly opposite effect compared with the aforementioned PDAC model. Thus, in different studies and tumor models, CAFs have a paradoxical effect in that their presence and activity can both stimulate and inhibit cancer growth and spread.

TGF- $\beta$ - which is secreted by cancer cells, CAFs, and inflammatory cells in the microenvironment is suspected to play a role in conferring this paradoxical effect related to CAFs. TGF- $\beta$ promotes fibroblast activation and aberrant ECM deposition (62); however, if TGF- $\beta$ signaling is eliminated, for example using a TGF- $\beta$ type II receptor-KO mouse, neoplasia of the stomach and prostate is initiated (63). As such, it would seem that TGF- $\beta$ is not wholly responsible for the paradoxical role that tumor fibroblasts play. RhoA has been proposed as a key regulator in the fibroblast transition from tumor inhibition to tumor promotion. RhoA-KO causes decreased $\alpha$-SMA expression, decreased fibroblast contractility, and increased tumor stiffness (64). SDF1 (CXCL12) of CAF origin is also known to be protumorigenic both by stimulating cancer cells directly via their CXCR4 receptor, as well as recruiting endothelial progenitors for tumor neoangiogenesis (65).

Which and in what circumstances CAFs are beneficial versus detrimental to tumors, and the phenotypic and signaling determinants of these seeming opposing roles, has yet to be fully elucidated. This is an active area of research inquiry. Continued discoveries on this topic are crucial toward developing therapies that effectively target tumor stroma with minimal would healing compromise.

\section{Acellular stromal elements of tumors and wounds}

In addition to the cells of the stroma, the protein and interstitial components of the ECM are increasingly recognized for their complex contribution to wound and tumor biology $(3,4,66)$. Overall, the former perception of the ECM as an inert structural framework over which autonomous cells enact biologic activities has been replaced by the recognition that the ECM is a dynamic manipulator of both wound healing and tumor behavior $(3,4,66)$.

ECM components in the stroma. Fibrin, formed by the breakdown of plasma fibrinogen and deposited into the ECM, is found early in matrix formation along with fibronectin and vitronectin (67). Fibroblasts recognize fibrin and then replace it with collagen during granulation tissue formation (68). With regard to tumor growth, Dvorak suggested a tumorigenic role of fibrin, proposing that it may aid tumorigenesis by providing scaffolding for malignant cells, shielding tumor cells from immune attack, and assisting with angiogenesis (69). Recent evidence shows that fibrin may have an even more active role in mediating malignant progression - fibrin-embedded lung tumor cells increase levels of fibronectin and activate integrins, leading to upregulation of Slug, an important mediator of EMT and metastases (70). The fibrin network also promotes angiogenesis in both tumor progression and wound healing.

Fibronectin also has a prominent role in stromal behavior. Fibronectin stretching leads to differential exposure of binding sites, which provides a wide spectrum of interactions with both cells and other stromal proteins $(3,71)$. In wound healing, in vitro studies have shown that fibronectin is required for collagen I deposition and maturation into fibrils (72). Fibronectin also regulates LOX, which is responsible for collagen covalent cross-linking (73). Additionally, fibronectin stimulates fibroblast expansion, migration, and contraction in a TGF- $\beta$-dependent manner (74-76). Similar to the wound healing processes, colon cancer CAFs promote cancer cell invasion in vitro in a process dependent on fibronectin fibrillogenesis and adhesion via $\alpha \mathrm{V} \beta 3$ integrins (77). Fibronectin is also implicated in tumor angiogenesis. Specifically, increased ECM stiffness induces alternative splicing to produce a splice variant containing extra domain-B (EDB) type III repeat, which favors tumor angiogenesis (78), again illustrating the complex relationship between mechanical properties of the matrix and cancer phenotypes.

Collagen is another ECM protein that significantly influences stroma biology. In wound healing, fibroblasts deposit collagen during the proliferative phase, which allows fibroblast adhesion and migration through the wound (3). Wound tissue becomes stiffer due to collagen deposition and cross-linking that effects fibroblast morphology and gene expression (79). TGF- $\beta$ is an important inducer of collagen synthesis in the wound, along with stimulating angiogenesis (80). Similar to wound healing, collagen affects tumors by modulation of the mechanical properties of the tumor bed. Human and mouse in vivo studies show that increased stiffness due to collagen thickening and linear conformation drive tumor invasion, mediated by LOX, FAK, and inte- 
grin clustering $(81,82)$. CAFs modulate the specific geometric profile of this collagen network via paracrine regulation of cross-linking, which affects tumor cell migration and invasiveness $(83,84)$. On the other hand, disrupting collagen cross-linking via LOX inhibition reduces tumor activity (82). LOX levels are clinically prognostic in head and neck cancer (85). Tumor compaction and LOX activation have also been linked to increased VEGF levels and angiogenesis in a glioblastoma mouse model (86). In vivo studies show that collagen signaling stabilizes expression of the EMT regulator SNAIL1 (87).

Integrins. Integrins are adhesion receptors that anchor cells to the ECM and transduce mechanical stimuli to effect outside-in and inside-out signaling mediated by FAK and Src tyrosine kinases $(66,88)$. Integrin expression profiles reflect the tensile state of wounds to promote closure. For example, $\alpha \mathrm{V} \beta 3$ integrin expression increases with higher wound tension and augments $\alpha$-SMA expression and cytoplasmic stress in fibroblasts in vitro (89). In tumors, integrins are crucial for cancer cell adhesion and invasion. Altering the TME fibrin network in vitro by adding platelet rich plasma modifies the integrin composition of the tumor stroma, which promotes tumor cell attachment (90). Additionally, integrin clustering is induced by tumor stiffness and leads to focal adhesion development. The downstream effects of this pathway include activation of cellular myosin by growth factors, as well as increased tumor cell proliferation, migration, metastases, and invasion $(82,91)$. Integrins are fundamental to CAF assembly of fibronectin networks to facilitate tumor invasion, especially integrin $\alpha \mathrm{V} \beta 3$ (77), which has been implicated in EMT and metastasis via Slug activation in vivo $(70,92)$. The local mechanism of tumor cell invasion is characterized by integrin attachment at the leading edge; conversely, proteolytic degradation of ECM fibers occurs at the trailing edge in vitro (93). Integrins are established activators of latent TGF- $\beta$ and present active TGF- $\beta$ to its cognate receptors via cell-cell contact (94-96). Finally, in vitro studies show that, when coupled with specific 3-D conformation of the TME, integrin upregulation switches on transcriptional factors required for tumor angiogenesis (97).

Interstitial glycans and glycosylated proteins. Hyaluronan (HA) is synthesized as a large glycosaminoglycan of repeating disaccharide and also exists in smaller fragments with distinct functions. In wound healing, high molecular weight HA leads to increased collagen III and decreased inflammation, while HA fragments increase inflammation and collagen I deposition, as well as myofibroblast differentiation and proliferation. Large pericellular HA molecules trap TGF- $\beta$ next to fibroblasts, resulting in a positive feedback loop to maintain the myofibroblast phenotype (98). Conversely, HA fragmentation appears to support tumor growth by increasing blood vessel recruitment (99), as well as metastasis via the transcription factor TWIST in human breast cancer cells (100). Clinically, high levels of HA expression convey a poor prognosis in breast cancer (101).

Decorin is a proteoglycan recognized for it's important role in regulating fibroblast signaling (102). Decorin is a high-affinity TGF- $\beta$ inhibitor (103) that substantially modulates stromal biology and has also been shown to regulate collagen fibrillogenesis and contraction $(104,105)$. Mouse decorin-KO models show delayed wound healing and increased fibroblast proliferation $(106,107)$. Like HA, decorin has a binary role in malignant spread: it is upregulated in quiescent, less aggressive forms of colon cancer (108), but $\mathrm{KO}$ can lead to intestinal tumor formation (109). In breast cancer, decorin downregulates ErbB2 tyrosine kinase signaling and blocks EGF, suppressing primary and metastatic progression (110, 111). However, high levels of decorin were also associated with lymph node disease and worsened survival with human proteomic profiling (112).

Protein-degrading ECM enzymes comprehensively modify stromal behavior in tumors and wounds, both by physical remodeling and regulation of proteins, cytokines, and proteases. Matrix metalloproteinases (MMPs), such as collagenase, gelatinase, and ADAMTS, modify the bioactivity of substrates, inducing changes in the interactions of these molecules with local cells and proteins (66). Early in wound healing, plasminogen is activated to plasmin to regulate fibrin degradation and fibrin matrix turnover, which influences downstream cellular behavior and protein deposition (113). In wound healing, MMPs facilitate cell migration, wound remodeling, and control bacterial invasion (114). This activity must be balanced, however, as overactive proteases are found in chronic wounds $(115,116)$. With regards to tumor progression, increased MMP activity confers invasive features to primary tumors (93). Microarray studies on human breast cancer show that MMP-9 is associated with more invasive tumors (117). Beyond the primary site, MMPs promote metastatic niches in lung cancer, and patients with lung metastases exhibit high levels of MMP-9 as compared with patients without distant disease (118). 


\section{The effect of immune cells within the tumor}

Immune cells are important components of the wound and TMEs, and immunotherapies, such as checkpoint inhibitors and adoptive $\mathrm{T}$ cell therapy, are actively being investigated. In wound healing, immune cells prevent infection while the skin barrier is breached. Additionally, immune cell signaling promotes wound closure by stimulating granulation and reepithelialization (119). The immune response to tumors has multiple and paradoxical roles, similar to fibroblasts and matrix proteins (120). It is an important defense mechanism against cancer growth (121); however, malignant transformation often occurs in chronically inflamed tissue that can support cancer growth $(122,123)$. Tumor cells can escape immune defenses by creating an immune-privileged site (121).

Several immune cell types present in the tumor stroma are specifically implicated in cancer progression. Tumor associated macrophages (TAMs) produce proangiogenic cytokines that support new vessel and tumor growth, recapitulating the proangiogenic actions of macrophages in the wound healing response (124). Both TAMs and cancer cells secrete IL-10, which suppresses killer T cell function and antitumor defenses $(125,126)$. In mouse mammary carcinoma studies, antibody blockade of a specific TAM scavenger receptor increased tumor immunogenicity, leading to decreased tumor progression and metastasis (127).

Cytotoxic and innate $\mathrm{T}$ cell responses are crucial in host efforts to halt tumor proliferation. Solid tumors are characterized by the presence or absence of a $\mathrm{T}$ cell infiltrate. Having a prominent intratumoral $\mathrm{T}$ cell infiltrate is associated with increased progression-free survival in gastric and colorectal cancer $(128,129)$. In the context of wound healing, ablation of cytotoxic $\mathrm{T}$ cells in mouse models changes the composition of the wound inflammatory infiltrate but does not impair wound closure, strength, and collagen deposition (130). Tregs and myleloid-derived suppressor cells may be triggered to act in protumorigenic manner by inhibiting protective killer T cell action (131). However, in wound healing, Tregs may promote wound healing by attenuating IFN- $\gamma$ and proinflammatory macrophage proliferation (132).

\section{Wounds that become tumors}

When cell stress is continuous and unrelenting, normal regenerative pathways can be derailed, and at times chronic wounds can ultimately result in neoplasia. Marjolin's ulcer describes an aggressive, ulcerating squamous cell carcinoma (SCC) in the setting of chronic wounds. Based on RNA-seq data, epithelial cells adjacent to Marjolin's ulcers show impaired ECM turnover, which may facilitate fibrosis and dysregulation of cell-cell adhesions related to EMT-associated cadherin signaling (133). Similarly, SCCs can form after tissue injury, such as actinic damage. Basal cell carcinoma (BCC) can arise from hair follicle cells secondary to the accumulation of mutations in follicular stem cells $(2,134)$. In a mouse skin biopsy model, BCClike tumors formed 10 weeks after injury, suggesting that BCC may be triggered by injury and subsequent migration of follicular stem cells. Various other types of tissue injury, from surgical incisions to stomach ulcers, are linked to cancer $(135,136)$.

\section{Tumor stroma and metastatic spread}

CAFs are involved in tumor progression through a variety of mechanisms, including paracrine signaling in the TME and ECM deposition (137). However, the extent to which CAFs actually pave the way for metastases is still unknown. Interestingly, CAFs express proteolytic enzymes that are involved in collagen IV degradation $(137,138)$. Collagen IV is a primary component of the basement membrane, degradation of which is a critical component for tumor invasion.

Less than $1 \%$ of the cancer cells that escape into circulation from a primary tumor site are actually able to establish themselves as metastatic tumors (139). Microenvironmental factors both at the primary tumor and metastatic sites are likely critical determinants of metastatic success. Tumor-derived cytokines are important in mobilizing supportive cells at distant sites (139). Some studies suggest that activation of local fibroblasts to form new, tissue-specific CAFs develop in the setting of metastasis. Duda et al. found that, in some circumstances, cancer cells might bring CAFs with them from the primary site, including into the brain in human disease (140). If cell surface marker expression is maintained in CAFs that travel from a primary to a metastatic site, then therapies targeting primary tumor stroma might be similarly effective in the setting of distant metastatic disease. 
Table 3. Clinical and preclinical stroma-targeting treatments

\section{Therapeutic targets}

PPAR $\gamma$ agonists

IFN- $\beta$-expressing MSCs (mesenchymal stem cells)

Receptor tyrosine kinase inhibitors (e.g., Imatinib)

AMD3100 (inhibits CAF CXCL12 production)

Anti-TGF- $\beta$ antibodies (to improve $\alpha$ PDL1 therapy)

Protease inhibitors

Anti-integrins

Antibodies to CAF cell surface markers (e.g., FAP- $\alpha$ and FSP1/S100A4 CAFs in invasive lobular carcinoma)

Modulation of PDCF-CC paracrine signaling

BMP signaling modulation

Hyaluronidase

\section{Role in tumor stroma}

PPAR $\gamma$ is a transcription factor that inhibits fibroblast proliferation in wound healing

(137). May also limit myofibroblast differentiation. Pioglitazone is a commercially

available PPAR $\gamma$ agonist being explored for prevention and treatment of gastrointestinal mucosal damage after radiation $(188,189)$ and for fibrosis prevention in nonalcoholic

fatty liver disease (NAFLD) (190). Suggested that Pioglitazone or other PPAR $\gamma$ agonists could have a role as an antistroma therapy.

The vitamin $\mathrm{D}$ receptor is expressed on human pancreatic tumor stroma. When treated with calcipotriol in preclinical studies, tumor stroma inflammation and fibrosis was decreased and pancreatic stellate fibroblasts reprogrammed back into a quiescent state (191).

In an ovarian cancer mouse model, i.p. injection of IFN- $\beta$-expressing MSCs (which

differentiated into $\alpha$-SMA-expressing myofibroblasts with activation), became

incorporated into tumors and killed cancer cells via IFN- $\beta$-mediated caspase-dependent apoptosis (192).

Imatinib is clinically used in the treatment of systemic fibrosis (143). Imatinib binds to the ATP-binding pocket of c-Abl, a downstream signaling molecule in the TCF- $\beta$

pathway, and also blocks the tyrosine kinase activity of PDGF receptors. In a cervical

cancer model, PDCF signaling blockade with Imatinib limited tumor growth.

In an endogenous mouse model of pancreatic ductal adenocarcinoma, when FAPexpressing CAF production of CXCL12 production was inhibited with administration of AMD3100, T cells could access the cancer cells and checkpoint inhibitor therapy ( $\alpha$ CTLA4 and $\alpha P D L 1$ ) became effective (144).

Administration of anti-TGF- $\beta$ antibodies improved response to $\alpha$ PDL1 therapy by inhibiting CAF TGF- $\beta$ signaling-mediated resistance to immunotherapy in a study on bladder cancer (145). Melanoma (147). Melanoma (147).

Using antibodies to block CAFs, such as by targeting cell surface markers associated with CAFs including FAP- $\alpha$ or FSP1/S100A4 CAFs in invasive lobular carcinoma (42). Modulation of PDCF-CC paracrine signaling in melanoma (19).

Based on the finding that BMP signaling is involved in myofibroblast lineage plasticity, altering the CAF phenotype could dramatically alter the tumor microenvironment in a way that might limit cancer cell proliferation and/or improve chemo-/immunotherapy efficacy (40).

Ongoing trials exploring the use of hyaluronidase in conjunction with standard chemotherapy in the setting of metastatic pancreatic cancer (146).

\section{Stroma as a therapeutic target}

CAFs regulate cancer cells and the tumor immune response, and as such, present an important therapeutic target in treating cancer (24). In contrast to cancer cells, the genetic stability of CAFs make them an excellent therapeutic target (141). Further, therapeutically weakening the stroma may promote better access of traditional chemotherapeutic or immunotherapeutic drugs to cancer cells. In PDAC, the prominence of stellate fibroblastic cells is thought to limit chemotherapy access to the tumor.

Existing medications hold potential for disrupting tumor stromal elements (Table 3). Imatinib, a tyrosine kinase inhibitor commonly used to treat leukemia, as well as gastrointestinal stromal tumors (GISTs), is being used for treatment of systemic fibrosis (142). Imatinib binds to the ATP-binding pocket of c-Abl, an important downstream signaling molecule of TGF- $\beta$. In c-Abl-null cells, TGF- $\beta$-dependent induction of ECM proteins is decreased. Imatinib also blocks the tyrosine kinase activity of PDGF receptors. In a gastric cancer model, PDGF signaling blockade with Imatinib limited tumor growth (143).

CAFs play an important role in regulating the response to immunotherapy. A mouse PDAC study showed that FAP-expressing CAFs inhibited $\mathrm{T}$ cell infiltration and the response to immune checkpoint inhibitors ( $\alpha$ CTLA4 and $\alpha$ PDL1). When SDF1 (CXCL12) production was inhibited with AMD3100, T cells accessed the cancer cells and checkpoint inhibitor therapy became effective (144). In a recent study exploring $\alpha$ PDL1 therapy in bladder cancer, fibroblast TGF- $\beta$ signaling was found to play a dramatic role in resistance to immunotherapy. Coadministration of $\alpha$ PDL1 and aTGF- $\beta$ antibodies increased therapeutic efficacy (145). Other ongoing trials are exploring the use of hyaluronidase, which targets tumor fibrosis, in 
conjunction with standard chemotherapy in metastatic PDAC (146). These studies highlight the potential importance of anti-CAF therapies to improve efficacy of current chemo- and immunotherapies.

In melanoma, several therapeutic strategies targeting the tumor stroma have been explored, including protease inhibitors, receptor tyrosine kinase inhibitors, and antiintegrins (147). Other avenues that hold potential for antistroma therapies include PDGF-CC paracrine signaling in melanoma (19), antibodies to FAP- $\alpha$ and FSP-1/S100A4 CAFs in invasive lobular carcinoma (42), and targets to stimulate BMP signaling. Therapies to stimulate fibroblast differentiation to adipose tissue may also modulate the TME to limit tumor proliferation and/or improve chemo-/immunotherapy response (40).

There are many potential difficulties or challenges associated with targeting the stroma. Because these cells resemble normal, nontransformed cells, finding specific targets that will not kill healthy fibroblasts systemically, and consequently have significant implications for wound healing, is difficult. One way around this could be to approach stromal therapies from the goal of "reeducating" stromal fibroblasts rather than ablating them (148). Candidate agents have yet to be broadly identified (39). This path will likely receive significantly more exploration in the coming years as our knowledge of tumor stroma, and parallels with wound healing pathways, continues to expand.

\section{Conclusion}

Activated fibroblasts in wound healing and CAFs share many of the same pathways but with distinct characteristics. These fibroblasts and their associated distinct and complex ECM have a primary role in tissue repair and tumor proliferation. In tumors, controlling CAFs proliferation and activities may limit tumor progression and improve response to therapies. However, a more complete understanding of the paradoxical role of fibroblasts in tumors is needed to fully harness this opportunity. As we learn more about the subtleties of the dynamic actions of the stroma, we expect that therapies will continue to be developed that modulate stroma in both wound healing and tumors. If a tumor cannot grow without establishing a microenvironment, then cancer proliferation and metastases cannot occur. With more investigators exploring the important cellular, molecular, and immunologic factors within the tumor stroma, and harnessing the vast knowledge regarding wound healing that shares clear corollaries in terms of the microenvironment, the probability of future novel and exciting antistroma therapies will increase.

\section{Author contributions}

DSF, REJ, and RCR reviewed the literature and prepared the manuscript. MTL and JAN oversaw the literature review and guided preparation and review of the manuscript. All authors reviewed and approved of the final manuscript.

\section{Acknowledgments}

The work was supported by the Hagey Laboratory for Pediatric Regenerative Medicine (DSF, REJ, RCR, MTL), The Gunn/Oliver Fund (DSF, REJ, RCR, MTL), American College of Surgeons Resident Research Fellowship (DSF), Advanced Residency Training at Stanford (ARTS) Program (DSF), Transplant and Tissue Engineering Center of Excellence Research Award Stanford University School of Medicine (REJ), and Stanford University School of Medicine Department of General Surgery (DSF, JAN).

Address correspondence to: Michael T. Longaker, 257 Campus Drive, Stanford, California 94305, USA. Phone: 650.736.1707; Email: longaker@stanford.edu. Or to: Jeffrey A. Norton, 875 Blake Wilbur Drive, Room CC-2230, Stanford, California 94305, USA. Phone: 650.498.6606; Email: janorton@stanford.edu.

1. Dvorak HF. Tumors: wounds that do not heal. Similarities between tumor stroma generation and wound healing. $N E n g l J M e d$. 1986;315(26):1650-1659.

2. Ge Y, et al. Stem Cell Lineage Infidelity Drives Wound Repair and Cancer. Cell. 2017;169(4):636-650.e14.

3. Tracy LE, Minasian RA, Caterson EJ. Extracellular Matrix and Dermal Fibroblast Function in the Healing Wound. Adv Wound Care (New Rochelle). 2016;5(3):119-136.

4. Lu P, Weaver VM, Werb Z. The extracellular matrix: a dynamic niche in cancer progression. J Cell Biol. 2012;196(4):395-406.

5. Pietras K, Pahler J, Bergers G, Hanahan D. Functions of paracrine PDGF signaling in the proangiogenic tumor stroma revealed by pharmacological targeting. PLoS Med. 2008;5(1):e19.

6. Öhlund D, Elyada E, Tuveson D. Fibroblast heterogeneity in the cancer wound. J Exp Med. 2014;211(8):1503-1523.

7. Rønnov-Jessen L, Petersen OW. Induction of alpha-smooth muscle actin by transforming growth factor-beta 1 in quiescent 
human breast gland fibroblasts. Implications for myofibroblast generation in breast neoplasia. Lab Invest. 1993;68(6):696-707.

8. Darby I, Skalli O, Gabbiani G. Alpha-smooth muscle actin is transiently expressed by myofibroblasts during experimental wound healing. Lab Invest. 1990;63(1):21-29.

9. Desmoulière A, Guyot C, Gabbiani G. The stroma reaction myofibroblast: a key player in the control of tumor cell behavior. Int J Dev Biol. 2004;48(5-6):509-517.

10. Strutz F, et al. Identification and characterization of a fibroblast marker: FSP1. J Cell Biol. 1995;130(2):393-405.

11. Eckes B, et al. Mechanical tension and integrin alpha 2 beta 1 regulate fibroblast functions. J Investig Dermatol Symp Proc. 2006;11(1):66-72.

12. Pierce GF, Mustoe TA, Altrock BW, Deuel TF, Thomason A. Role of platelet-derived growth factor in wound healing. $J$ Cell Biochem. 1991;45(4):319-326.

13. Wang X, et al. Feedback Activation of Basic Fibroblast Growth Factor Signaling via the Wnt/ $\beta$-Catenin Pathway in Skin Fibroblasts. Front Pharmacol. 2017;8:32.

14. Brennen WN, Isaacs JT, Denmeade SR. Rationale behind targeting fibroblast activation protein-expressing carcinoma-associated fibroblasts as a novel chemotherapeutic strategy. Mol Cancer Ther. 2012;11(2):257-266.

15. Akhmetshina A, et al. Activation of canonical Wnt signalling is required for TGF- $\beta$-mediated fibrosis. Nat Commun. 2012;3:735

16. Philippeos C, et al. Spatial and Single-Cell Transcriptional Profiling Identifies Functionally Distinct Human Dermal Fibroblast Subpopulations. J Invest Dermatol. 2018;138(4):811-825.

17. Liu S, et al. Expression of integrin beta1 by fibroblasts is required for tissue repair in vivo. J Cell Sci. 2010;123(Pt 21):3674-3682.

18. Pakyari M, Farrokhi A, Maharlooei MK, Ghahary A. Critical Role of Transforming Growth Factor Beta in Different Phases of Wound Healing. Adv Wound Care (New Rochelle). 2013;2(5):215-224.

19. Anderberg C, et al. Paracrine signaling by platelet-derived growth factor-CC promotes tumor growth by recruitment of cancer-associated fibroblasts. Cancer Res. 2009;69(1):369-378.

20. Ruffini F, et al. Platelet-derived growth factor-C promotes human melanoma aggressiveness through activation of neuropilin-1. Oncotarget. 2017;8(40):66833-66848.

21. Poser I, Domínguez D, de Herreros AG, Varnai A, Buettner R, Bosserhoff AK. Loss of E-cadherin expression in melanoma cells involves up-regulation of the transcriptional repressor Snail. J Biol Chem. 2001;276(27):24661-24666.

22. Hsu M, Andl T, Li G, Meinkoth JL, Herlyn M. Cadherin repertoire determines partner-specific gap junctional communication during melanoma progression. J Cell Sci. 2000;113(Pt 9):1535-1542.

23. Zeisberg EM, Zeisberg M. The role of promoter hypermethylation in fibroblast activation and fibrogenesis. $J$ Pathol. 2013;229(2):264-273.

24. Shiga K, Hara M, Nagasaki T, Sato T, Takahashi H, Takeyama H. Cancer-Associated Fibroblasts: Their Characteristics and Their Roles in Tumor Growth. Cancers (Basel). 2015;7(4):2443-2458.

25. Chang HY, et al. Gene expression signature of fibroblast serum response predicts human cancer progression: similarities between tumors and wounds. PLoS Biol. 2004;2(2):E7.

26. Desmoulière A, Redard M, Darby I, Gabbiani G. Apoptosis mediates the decrease in cellularity during the transition between granulation tissue and scar. Am J Pathol. 1995;146(1):56-66

27. Amornsupak K, et al. High ASMA+ Fibroblasts and Low Cytoplasmic HMGB1+ Breast Cancer Cells Predict Poor Prognosis. Clin Breast Cancer. 2017;17(6):441-452.e2.

28. Hawinkels LJ, et al. Interaction with colon cancer cells hyperactivates TGF- $\beta$ signaling in cancer-associated fibroblasts. Oncogene. 2014;33(1):97-107.

29. Wendling O, Bornert JM, Chambon P, Metzger D. Efficient temporally-controlled targeted mutagenesis in smooth muscle cells of the adult mouse. Genesis. 2009;47(1):14-18.

30. Erez N, Truitt M, Olson P, Arron ST, Hanahan D. Cancer-Associated Fibroblasts Are Activated in Incipient Neoplasia to Orchestrate Tumor-Promoting Inflammation in an NF-kappaB-Dependent Manner. Cancer Cell. 2010;17(2):135-147.

31. Sugimoto H, Mundel TM, Kieran MW, Kalluri R. Identification of fibroblast heterogeneity in the tumor microenvironment Cancer Biol Ther. 2006;5(12):1640-1646.

32. Xin Y, et al. Expansion of CD26 positive fibroblast population promotes keloid progression. Exp Cell Res. 2017;356(1):104-113.

33. Rinkevich Y, et al. Skin fibrosis. Identification and isolation of a dermal lineage with intrinsic fibrogenic potential. Science. 2015;348(6232):aaa2151.

34. Walmsley GG, et al. Scarless wound healing: chasing the holy grail. Plast Reconstr Surg. 2015;135(3):907-917.

35. Larjava H, Wiebe C, Gallant-Behm C, Hart DA, Heino J, Häkkinen L. Exploring scarless healing of oral soft tissues. J Can Dent Assoc. 2011;77:b18.

36. Cheng F, et al. Vimentin coordinates fibroblast proliferation and keratinocyte differentiation in wound healing via TGF- $\beta$-Slug signaling. Proc Natl Acad Sci USA. 2016;113(30):E4320-E4327.

37. Yoshida T, Akatsuka T, Imanaka-Yoshida K. Tenascin-C and integrins in cancer. Cell Adh Migr. 2015;9(1-2):96-104.

38. Kikuchi Y, et al. Periostin is expressed in pericryptal fibroblasts and cancer-associated fibroblasts in the colon. $J$ Histochem Cytochem. 2008;56(8):753-764.

39. Kalluri R. The biology and function of fibroblasts in cancer. Nat Rev Cancer. 2016;16(9):582-598.

40. Plikus MV, et al. Regeneration of fat cells from myofibroblasts during wound healing. Science. 2017;355(6326):748-752.

41. Shiga K, Hara M, Nagasaki T, Sato T, Takahashi H, Takeyama H. Cancer-Associated Fibroblasts: Their Characteristics and Their Roles in Tumor Growth. Cancers (Basel). 2015;7(4):2443-2458.

42. Park CK, Jung WH, Koo JS. Expression of cancer-associated fibroblast-related proteins differs between invasive lobular carcinoma and invasive ductal carcinoma. Breast Cancer Res Treat. 2016;159(1):55-69.

43. Wikberg ML, et al. High intratumoral expression of fibroblast activation protein (FAP) in colon cancer is associated with poorer patient prognosis. Tumour Biol. 2013;34(2):1013-1020.

44. Ishii G, et al. Bone-marrow-derived myofibroblasts contribute to the cancer-induced stromal reaction. Biochem Biophys Res Commun. 2003;309(1):232-240.

45. Elenbaas B, et al. Human breast cancer cells generated by oncogenic transformation of primary mammary epithelial cells. Genes 
Dev. 2001;15(1):50-65.

46. Orimo A, et al. Stromal fibroblasts present in invasive human breast carcinomas promote tumor growth and angiogenesis through elevated SDF-1/CXCL12 secretion. Cell. 2005;121(3):335-348.

47. Alba-Castellón L, et al. Snail1-Dependent Activation of Cancer-Associated Fibroblast Controls Epithelial Tumor Cell Invasion and Metastasis. Cancer Res. 2016;76(21):6205-6217.

48. Giraudo E, Inoue M, Hanahan D. An amino-bisphosphonate targets MMP-9-expressing macrophages and angiogenesis to impair cervical carcinogenesis. J Clin Invest. 2004;114(5):623-633.

49. Lin ZQ, Kondo T, Ishida Y, Takayasu T, Mukaida N. Essential involvement of IL-6 in the skin wound-healing process as evidenced by delayed wound healing in IL-6-deficient mice. J Leukoc Biol. 2003;73(6):713-721.

50. Hill R, Song Y, Cardiff RD, Van Dyke T. Selective evolution of stromal mesenchyme with p53 loss in response to epithelial tumorigenesis. Cell. 2005;123(6):1001-1011.

51. Patocs A, et al. Breast-cancer stromal cells with TP53 mutations and nodal metastases. N Engl J Med. 2007;357(25):2543-2551.

52. Hawsawi NM, et al. Breast carcinoma-associated fibroblasts and their counterparts display neoplastic-specific changes. Cancer Res. 2008;68(8):2717-2725.

53. Bar J, Moskovits N, Oren M. Involvement of stromal p53 in tumor-stroma interactions. Semin Cell Dev Biol. 2010;21(1):47-54.

54. Addadi Y, et al. p53 status in stromal fibroblasts modulates tumor growth in an SDF1-dependent manner. Cancer Res. 2010;70(23):9650-9658.

55. Otomo R, et al. TSPAN12 is a critical factor for cancer-fibroblast cell contact-mediated cancer invasion. Proc Natl Acad Sci USA. 2014;111(52):18691-18696.

56. Lujambio A, et al. Non-cell-autonomous tumor suppression by p53. Cell. 2013;153(2):449-460

57. Mueller MM, Fusenig NE. Friends or foes - bipolar effects of the tumour stroma in cancer. Nat Rev Cancer. 2004;4(11):839-849.

58. Özdemir BC, et al. Depletion of carcinoma-associated fibroblasts and fibrosis induces immunosuppression and accelerates pancreas cancer with reduced survival. Cancer Cell. 2014;25(6):719-734.

59. Paulsson J, Micke P. Prognostic relevance of cancer-associated fibroblasts in human cancer. Semin Cancer Biol. 2014;25:61-68.

60. McMillin DW, Negri JM, Mitsiades CS. The role of tumour-stromal interactions in modifying drug response: challenges and opportunities. Nat Rev Drug Discov. 2013;12(3):217-228.

61. Cohen $\mathrm{N}$, et al. Fibroblasts drive an immunosuppressive and growth-promoting microenvironment in breast cancer via secretion of Chitinase 3-like 1. Oncogene. 2017;36(31):4457-4468.

62. Margadant C, Sonnenberg A. Integrin-TGF-beta crosstalk in fibrosis, cancer and wound healing. EMBO Rep. 2010;11(2):97-105.

63. Bhowmick NA, et al. TGF-beta signaling in fibroblasts modulates the oncogenic potential of adjacent epithelia. Science. 2004;303(5659):848-851.

64. Alkasalias T, et al. RhoA knockout fibroblasts lose tumor-inhibitory capacity in vitro and promote tumor growth in vivo. Proc Natl Acad Sci USA. 2017;114(8):E1413-E1421.

65. Orimo A, Weinberg RA. Stromal fibroblasts in cancer: a novel tumor-promoting cell type. Cell Cycle. 2006;5(15):1597-1601.

66. Theocharis AD, Skandalis SS, Gialeli C, Karamanos NK. Extracellular matrix structure. Adv Drug Deliv Rev. 2016;97:4-27.

67. Miron RJ, Fujioka-Kobayashi M, Bishara M, Zhang Y, Hernandez M, Choukroun J. Platelet-Rich Fibrin and Soft Tissue Wound Healing: A Systematic Review. Tissue Eng Part B Rev. 2017;23(1):83-99.

68. Tuan TL, Song A, Chang S, Younai S, Nimni ME. In vitro fibroplasia: matrix contraction, cell growth, and collagen production of fibroblasts cultured in fibrin gels. Exp Cell Res. 1996;223(1):127-134.

69. Dvorak HF, Senger DR, Dvorak AM. Fibrin as a component of the tumor stroma: origins and biological significance. Cancer Metastasis Rev. 1983;2(1):41-73.

70. Knowles LM, Gurski LA, Engel C, Gnarra JR, Maranchie JK, Pilch J. Integrin av $\beta 3$ and fibronectin upregulate Slug in cancer cells to promote clot invasion and metastasis. Cancer Res. 2013;73(20):6175-6184.

71. Klotzsch E, et al. Fibronectin forms the most extensible biological fibers displaying switchable force-exposed cryptic binding sites. Proc Natl Acad Sci USA. 2009;106(43):18267-18272.

72. Sottile J, Hocking DC. Fibronectin polymerization regulates the composition and stability of extracellular matrix fibrils and cell-matrix adhesions. Mol Biol Cell. 2002;13(10):3546-3559

73. Fogelgren B, et al. Cellular fibronectin binds to lysyl oxidase with high affinity and is critical for its proteolytic activation. J Biol Chem. 2005;280(26):24690-24697.

74. Gildner CD, Roy DC, Farrar CS, Hocking DC. Opposing effects of collagen I and vitronectin on fibronectin fibril structure and function. Matrix Biol. 2014;34:33-45.

75. Obara M, Yoshizato K. A novel domain of fibronectin revealed by epitope mapping of a monoclonal antibody which inhibits fibroblasts-mediated collagen gel contraction. FEBS Lett. 1997;412(1):48-52.

76. Corbett SA, Lee L, Wilson CL, Schwarzbauer JE. Covalent cross-linking of fibronectin to fibrin is required for maximal cell adhesion to a fibronectin-fibrin matrix. J Biol Chem. 1997;272(40):24999-25005.

77. Attieh Y, et al. Cancer-associated fibroblasts lead tumor invasion through integrin- $\beta 3$-dependent fibronectin assembly. $J$ Cell Biol. 2017;216(11):3509-3520.

78. Bordeleau F, et al. Tissue stiffness regulates serine/arginine-rich protein-mediated splicing of the extra domain B-fibronectin isoform in tumors. Proc Natl Acad Sci USA. 2015;112(27):8314-8319.

79. Liu F, et al. Feedback amplification of fibrosis through matrix stiffening and COX-2 suppression. J Cell Biol. 2010;190(4):693-706

80. Roberts AB, et al. Transforming growth factor type beta: rapid induction of fibrosis and angiogenesis in vivo and stimulation of collagen formation in vitro. Proc Natl Acad Sci USA. 1986;83(12):4167-4171.

81. Erler JT, et al. Lysyl oxidase is essential for hypoxia-induced metastasis. Nature. 2006;440(7088):1222-1226.

82. Levental KR, et al. Matrix crosslinking forces tumor progression by enhancing integrin signaling. Cell. 2009;139(5):891-906

83. Pankova D, et al. Cancer-Associated Fibroblasts Induce a Collagen Cross-link Switch in Tumor Stroma. Mol Cancer Res. 2016;14(3):287-295.

84. Chen Y, et al. Lysyl hydroxylase 2 induces a collagen cross-link switch in tumor stroma. J Clin Invest. 2015;125(3):1147-1162.

85. Le QT, et al. Validation of lysyl oxidase as a prognostic marker for metastasis and survival in head and neck squamous cell car- 
cinoma: Radiation Therapy Oncology Group trial 90-03. J Clin Oncol. 2009;27(26):4281-4286.

86. Mammoto T, Jiang A, Jiang E, Panigrahy D, Kieran MW, Mammoto A. Role of collagen matrix in tumor angiogenesis and glioblastoma multiforme progression. Am J Pathol. 2013;183(4):1293-1305.

87. Zhang K, et al. The collagen receptor discoidin domain receptor 2 stabilizes SNAIL1 to facilitate breast cancer metastasis. Nat Cell Biol. 2013;15(6):677-687.

88. Schnittert J, Bansal R, Storm G, Prakash J. Integrins in wound healing, fibrosis and tumor stroma: High potential targets for therapeutics and drug delivery. Adv Drug Deliv Rev. 2018;129:37-53.

89. Jones C, Ehrlich HP. Fibroblast expression of $\alpha$-smooth muscle actin, $\alpha 2 \beta 1$ integrin and $\alpha v \beta 3$ integrin: influence of surface rigidity. Exp Mol Pathol. 2011;91(1):394-399.

90. Andrade SS, et al. Interface between breast cancer cells and the tumor microenvironment using platelet-rich plasma to promote tumor angiogenesis - influence of platelets and fibrin bundles on the behavior of breast tumor cells. Oncotarget. 2017;8(10):16851-16874.

91. Paszek MJ, et al. Tensional homeostasis and the malignant phenotype. Cancer Cell. 2005;8(3):241-254.

92. Kaplan RN, et al. VEGFR1-positive haematopoietic bone marrow progenitors initiate the pre-metastatic niche. Nature. 2005;438(7069):820-827.

93. Wolf K, et al. Multi-step pericellular proteolysis controls the transition from individual to collective cancer cell invasion. Nat Cell Biol. 2007;9(8):893-904.

94. Conroy KP, Kitto LJ, Henderson NC. av integrins: key regulators of tissue fibrosis. Cell Tissue Res. 2016;365(3):511-519.

95. Munger JS, et al. The integrin alpha v beta 6 binds and activates latent TGF beta 1: a mechanism for regulating pulmonary inflammation and fibrosis. Cell. 1999;96(3):319-328.

96. Prieto AL, Edelman GM, Crossin KL. Multiple integrins mediate cell attachment to cytotactin/tenascin. Proc Natl Acad Sci USA. 1993;90(21):10154-10158.

97. Velez DO, et al. 3D collagen architecture induces a conserved migratory and transcriptional response linked to vasculogenic mimicry. Nat Commun. 2017;8(1):1651

98. Webber J, Meran S, Steadman R, Phillips A. Hyaluronan orchestrates transforming growth factor-beta1-dependent maintenance of myofibroblast phenotype. J Biol Chem. 2009;284(14):9083-9092.

99. Koyama H, et al. Hyperproduction of hyaluronan in neu-induced mammary tumor accelerates angiogenesis through stromal cell recruitment: possible involvement of versican/PG-M. Am J Pathol. 2007;170(3):1086-1099.

100. Bourguignon LY, Wong G, Earle C, Krueger K, Spevak CC. Hyaluronan-CD44 interaction promotes c-Src-mediated twist signaling, microRNA-10b expression, and RhoA/RhoC up-regulation, leading to Rho-kinase-associated cytoskeleton activation and breast tumor cell invasion. J Biol Chem. 2010;285(47):36721-36735.

101. Auvinen P, et al. Increased hyaluronan content and stromal cell CD44 associate with HER2 positivity and poor prognosis in human breast cancer. Int J Cancer. 2013;132(3):531-539.

102. Mohan RR, Gupta R, Mehan MK, Cowden JW, Sinha S. Decorin transfection suppresses profibrogenic genes and myofibroblast formation in human corneal fibroblasts. Exp Eye Res. 2010;91(2):238-245.

103. Yamaguchi Y, Mann DM, Ruoslahti E. Negative regulation of transforming growth factor-beta by the proteoglycan decorin Nature. 1990;346(6281):281-284.

104. Chen S, Young MF, Chakravarti S, Birk DE. Interclass small leucine-rich repeat proteoglycan interactions regulate collagen fibrillogenesis and corneal stromal assembly. Matrix Biol. 2014;35:103-111.

105. Bittner K, Liszio C, Blumberg P, Schönherr E, Kresse H. Modulation of collagen gel contraction by decorin. Biochem J. 1996;314(Pt 1):159-166.

106. Järveläinen H, et al. A role for decorin in cutaneous wound healing and angiogenesis. Wound Repair Regen. 2006;14(4):443-452.

107. Ferdous Z, Peterson SB, Tseng H, Anderson DK, Iozzo RV, Grande-Allen KJ. A role for decorin in controlling proliferation, adhesion, and migration of murine embryonic fibroblasts. J Biomed Mater Res A. 2010;93(2):419-428.

108. Santra M, Skorski T, Calabretta B, Lattime EC, Iozzo RV. De novo decorin gene expression suppresses the malignant phenotype in human colon cancer cells. Proc Natl Acad Sci USA. 1995;92(15):7016-7020.

109. Bi X, et al. Genetic deficiency of decorin causes intestinal tumor formation through disruption of intestinal cell maturation. Carcinogenesis. 2008;29(7):1435-1440.

110. Reed CC, et al. Decorin prevents metastatic spreading of breast cancer. Oncogene. 2005;24(6):1104-1110.

111. Seidler DG, et al. Decorin protein core inhibits in vivo cancer growth and metabolism by hindering epidermal growth factor receptor function and triggering apoptosis via caspase-3 activation. J Biol Chem. 2006;281(36):26408-26418.

112. Cawthorn TR, et al. Proteomic analyses reveal high expression of decorin and endoplasmin (HSP90B1) are associated with breast cancer metastasis and decreased survival. PLOS ONE. 2012;7(2):e30992.

113. Law RH, Abu-Ssaydeh D, Whisstock JC. New insights into the structure and function of the plasminogen/plasmin system. Curr Opin Struct Biol. 2013;23(6):836-841.

114. Cole AM, Shi J, Ceccarelli A, Kim YH, Park A, Ganz T. Inhibition of neutrophil elastase prevents cathelicidin activation and impairs clearance of bacteria from wounds. Blood. 2001;97(1):297-304.

115. Yager DR, Zhang LY, Liang HX, Diegelmann RF, Cohen IK. Wound fluids from human pressure ulcers contain elevated matrix metalloproteinase levels and activity compared to surgical wound fluids. J Invest Dermatol. 1996;107(5):743-748.

116. Rayment EA, Upton Z, Shooter GK. Increased matrix metalloproteinase-9 (MMP-9) activity observed in chronic wound fluid is related to the clinical severity of the ulcer. Br J Dermatol. 2008;158(5):951-961.

117. Merdad A, et al. Expression of matrix metalloproteinases (MMPs) in primary human breast cancer: MMP-9 as a potential biomarker for cancer invasion and metastasis. Anticancer Res. 2014;34(3):1355-1366.

118. Hiratsuka S, et al. MMP9 induction by vascular endothelial growth factor receptor-1 is involved in lung-specific metastasis. Cancer Cell. 2002;2(4):289-300.

119. Shaw TJ, Martin P. Wound repair: a showcase for cell plasticity and migration. Curr Opin Cell Biol. 2016;42:29-37.

120. Schreiber RD, Old LJ, Smyth MJ. Cancer immunoediting: integrating immunity's roles in cancer suppression and promotion. Science. 2011;331(6024):1565-1570. 
121. Joyce JA, Fearon DT. T cell exclusion, immune privilege, and the tumor microenvironment. Science. 2015;348(6230):74-80.

122. Balkwill F, Mantovani A. Inflammation and cancer: back to Virchow? Lancet. 2001;357(9255):539-545.

123. Virchow R. Cellular Pathology: Physiological and Pathological Histology. Biodiversity Heritage Library. https://www.biodiversitylibrary.org/item/77126\#page/1/mode/1up. Accessed September 11, 2018.

124. Lucas T, et al. Differential roles of macrophages in diverse phases of skin repair. J Immunol. 2010;184(7):3964-3977.

125. Coussens LM, Werb Z. Inflammation and cancer. Nature. 2002;420(6917):860-867.

126. Torisu $\mathrm{H}$, et al. Macrophage infiltration correlates with tumor stage and angiogenesis in human malignant melanoma: possible involvement of TNFalpha and IL-1alpha. Int J Cancer. 2000;85(2):182-188.

127. Georgoudaki AM, et al. Reprogramming Tumor-Associated Macrophages by Antibody Targeting Inhibits Cancer Progression and Metastasis. Cell Rep. 2016;15(9):2000-2011.

128. Gajewski TF, Schreiber H, Fu YX. Innate and adaptive immune cells in the tumor microenvironment. Nat Immunol. 2013;14(10):1014-1022.

129. Rusakiewicz S, et al. Immune infiltrates are prognostic factors in localized gastrointestinal stromal tumors. Cancer Res. 2013;73(12):3499-3510.

130. Chen L, Mehta ND, Zhao Y, DiPietro LA. Absence of CD4 or CD8 lymphocytes changes infiltration of inflammatory cells and profiles of cytokine expression in skin wounds, but does not impair healing. Exp Dermatol. 2014;23(3):189-194.

131. Chaudhary B, Elkord E. Regulatory T Cells in the Tumor Microenvironment and Cancer Progression: Role and Therapeutic Targeting. Vaccines (Basel). 2016;4(3):28.

132. Nosbaum A, et al. Cutting Edge: Regulatory T Cells Facilitate Cutaneous Wound Healing. J Immunol. 2016;196(5):2010-2014.

133. Sinha S, et al. Transcriptional Analysis Reveals Evidence of Chronically Impeded ECM Turnover and Epithelium-to-Mesenchyme Transition in Scar Tissue Giving Rise to Marjolin's Ulcer. J Burn Care Res. 2017;38(1):e14-e22.

134. Donati G, et al. Wounding induces dedifferentiation of epidermal Gata6 + cells and acquisition of stem cell properties. Nat Cell Biol. 2017;19(6):603-613.

135. Hansson LE, et al. The risk of stomach cancer in patients with gastric or duodenal ulcer disease. $N$ Engl J Med. 1996;335(4):242-249.

136. Corbo MD, O'Malley M. Marjolin ulcer in a surgical scar. Cutis. 2015;95(5):E8-E10.

137. Rybinski B, Franco-Barraza J, Cukierman E. The wound healing, chronic fibrosis, and cancer progression triad. Physiol Genomics. 2014;46(7):223-244.

138. Maneva-Radicheva L, Ebert U, Dimoudis N, Altankov G. Fibroblast remodeling of adsorbed collagen type IV is altered in contact with cancer cells. Histol Histopathol. 2008;23(7):833-842.

139. McAllister SS, Weinberg RA. The tumour-induced systemic environment as a critical regulator of cancer progression and metastasis. Nat Cell Biol. 2014;16(8):717-727.

140. Duda DG, et al. Malignant cells facilitate lung metastasis by bringing their own soil. Proc Natl Acad Sci USA. 2010;107(50):21677-21682.

141. Palumbo A, Da Costa Nde O, Bonamino MH, Pinto LF, Nasciutti LE. Genetic instability in the tumor microenvironment: a new look at an old neighbor. Mol Cancer. 2015;14:145.

142. Fraticelli P, et al. Low-dose oral imatinib in the treatment of systemic sclerosis interstitial lung disease unresponsive to cyclophosphamide: a phase II pilot study. Arthritis Res Ther. 2014;16(4):R144.

143. Sumida T, et al. Anti-stromal therapy with imatinib inhibits growth and metastasis of gastric carcinoma in an orthotopic nude mouse model. Int J Cancer. 2011;128(9):2050-2062.

144. Feig C, et al. Targeting CXCL12 from FAP-expressing carcinoma-associated fibroblasts synergizes with anti-PD-L1 immunotherapy in pancreatic cancer. Proc Natl Acad Sci USA. 2013;110(50):20212-20217.

145. Mariathasan S, et al. TGF $\beta$ attenuates tumour response to PD-L1 blockade by contributing to exclusion of T cells. Nature. 2018;554(7693):544-548.

146. A Study of PEGylated Recombinant Human Hyaluronidase in Combination With Nab-Paclitaxel Plus Gemcitabine Compared With Placebo Plus Nab-Paclitaxel and Gemcitabine in Participants With Hyaluronan-High Stage IV Previously Untreated Pancreatic Ductal Adenocarcinoma. ClinicalTrials.gov. https://clinicaltrials.gov/ct2/show/NCT02715804. Published March 22, 2016. Updated July 27, 2018. Accessed August 30, 2018.

147. Smalley KS, Lioni M, Herlyn M. Targeting the stromal fibroblasts: a novel approach to melanoma therapy. Expert Rev Anticancer Ther. 2005;5(6):1069-1078.

148. Quail DF, Joyce JA. Microenvironmental regulation of tumor progression and metastasis. Nat Med. 2013;19(11):1423-1437.

149. Hinz B, Celetta G, Tomasek JJ, Gabbiani G, Chaponnier C. Alpha-smooth muscle actin expression upregulates fibroblast contractile activity. Mol Biol Cell. 2001;12(9):2730-2741.

150.Ibrahim MM, et al. Myofibroblasts contribute to but are not necessary for wound contraction. Lab Invest. 2015;95(12):1429-1438

151. Garin-Chesa P, Old LJ, Rettig WJ. Cell surface glycoprotein of reactive stromal fibroblasts as a potential antibody target in human epithelial cancers. Proc Natl Acad Sci USA. 1990;87(18):7235-7239.

152. Opalenik SR, Davidson JM. Fibroblast differentiation of bone marrow-derived cells during wound repair. FASEB J. 2005;19(11):1561-1563.

153. Grum-Schwensen B, et al. Suppression of tumor development and metastasis formation in mice lacking the S100A4(mts1) gene Cancer Res. 2005;65(9):3772-3780.

154. Marsh T, Pietras K, McAllister SS. Fibroblasts as architects of cancer pathogenesis. Biochim Biophys Acta. 2013;1832(7):1070-1078.

155. Walker N, et al. Resident tissue-specific mesenchymal progenitor cells contribute to fibrogenesis in human lung allografts. $A m J$ Pathol. 2011;178(6):2461-2469.

156. Arnold SA, Loomans HA, Ketova T, Andl CD, Clark PE, Zijlstra A. Urinary oncofetal ED-A fibronectin correlates with poor prognosis in patients with bladder cancer. Clin Exp Metastasis. 2016;33(1):29-44.

157. Mao Y, Keller ET, Garfield DH, Shen K, Wang J. Stromal cells in tumor microenvironment and breast cancer. Cancer Metastasis Rev. 2013;32(1-2):303-315.

158. Walker JT, McLeod K, Kim S, Conway SJ, Hamilton DW. Periostin as a multifunctional modulator of the wound healing 
response. Cell Tissue Res. 2016;365(3):453-465.

159. Fukushima N, Kikuchi Y, Nishiyama T, Kudo A, Fukayama M. Periostin deposition in the stroma of invasive and intraductal neoplasms of the pancreas. Mod Pathol. 2008;21(8):1044-1053.

160. Skalli O, et al. Myofibroblasts from diverse pathologic settings are heterogeneous in their content of actin isoforms and intermediate filament proteins. Lab Invest. 1989;60(2):275-285.

161. Nazari B, et al. Altered Dermal Fibroblasts in Systemic Sclerosis Display Podoplanin and CD90. Am J Pathol. 2016;186(10):2650-2664.

162. Kawase A, et al. Podoplanin expression by cancer associated fibroblasts predicts poor prognosis of lung adenocarcinoma. Int $J$ Cancer. 2008;123(5):1053-1059.

163. Ono S, et al. Podoplanin-positive cancer-associated fibroblasts could have prognostic value independent of cancer cell phenotype in stage I lung squamous cell carcinoma: usefulness of combining analysis of both cancer cell phenotype and cancer-associated fibroblast phenotype. Chest. 2013;143(4):963-970.

164. Kalluri R, Zeisberg M. Fibroblasts in cancer. Nat Rev Cancer. 2006;6(5):392-401.

165. Tabib T, Morse C, Wang T, Chen W, Lafyatis R. SFRP2/DPP4 and FMO1/LSP1 Define Major Fibroblast Populations in Human Skin. J Invest Dermatol. 2018;138(4):802-810.

166. Driskell RR, et al. Distinct fibroblast lineages determine dermal architecture in skin development and repair. Nature. 2013;504(7479):277-281.

167. Ishii G, Ochiai A, Neri S. Phenotypic and functional heterogeneity of cancer-associated fibroblast within the tumor microenvironment. Adv Drug Deliv Rev. 2016;99(Pt B):186-196.

168. Sardone F, et al. Collagen VI-NG2 axis in human tendon fibroblasts under conditions mimicking injury response. Matrix Biol. 2016;55:90-105.

169. Silini A, et al. Regulator of G-protein signaling 5 (RGS5) protein: a novel marker of cancer vasculature elicited and sustained by the tumor's proangiogenic microenvironment. Cell Mol Life Sci. 2012;69(7):1167-1178.

170. Trebaul A, Chan EK, Midwood KS. Regulation of fibroblast migration by tenascin-C. Biochem Soc Trans. 2007;35(Pt 4):695-697.

171. Yu Y, Xiao CH, Tan LD, Wang QS, Li XQ, Feng YM. Cancer-associated fibroblasts induce epithelial-mesenchymal transition of breast cancer cells through paracrine TGF- $\beta$ signalling. Br J Cancer. 2014;110(3):724-732.

172. Calon A, et al. Stromal gene expression defines poor-prognosis subtypes in colorectal cancer. Nat Genet. 2015;47(4):320-329.

173. Quante M, et al. Bone marrow-derived myofibroblasts contribute to the mesenchymal stem cell niche and promote tumor growth. Cancer Cell. 2011;19(2):257-272.

174. Tyan SW, et al. Breast cancer cells induce cancer-associated fibroblasts to secrete hepatocyte growth factor to enhance breast tumorigenesis. PLoS One. 2011;6(1):e15313.

175. Wilson TR, et al. Widespread potential for growth-factor-driven resistance to anticancer kinase inhibitors. Nature. 2012;487(7408):505-509.

176. Wang W, et al. Crosstalk to stromal fibroblasts induces resistance of lung cancer to epidermal growth factor receptor tyrosine kinase inhibitors. Clin Cancer Res. 2009;15(21):6630-6638.

177. Dong J, et al. VEGF-null cells require PDGFR alpha signaling-mediated stromal fibroblast recruitment for tumorigenesis. EMBO J. 2004;23(14):2800-2810.

178. Lewis DA, Travers JB, Somani AK, Spandau DF. The IGF-1/IGF-1R signaling axis in the skin: a new role for the dermis in aging-associated skin cancer. Oncogene. 2010;29(10):1475-1485.

179. Wu X, et al. IL-6 secreted by cancer-associated fibroblasts promotes epithelial-mesenchymal transition and metastasis of gastric cancer via JAK2/STAT3 signaling pathway. Oncotarget. 2017;8(13):20741-20750.

180. Wang T, et al. Senescent Carcinoma-Associated Fibroblasts Upregulate IL8 to Enhance Prometastatic Phenotypes. Mol Cancer Res. 2017;15(1):3-14.

181. Razmkhah M, Jaberipour M, Hosseini A, Safaei A, Khalatbari B, Ghaderi A. Expression profile of IL-8 and growth factors in breast cancer cells and adipose-derived stem cells (ASCs) isolated from breast carcinoma. Cell Immunol. 2010;265(1):80-85.

182. Chaturvedi $\mathrm{P}$, et al. Hypoxia-inducible factor-dependent breast cancer-mesenchymal stem cell bidirectional signaling promotes metastasis. J Clin Invest. 2013;123(1):189-205.

183. Harper J, Sainson RC. Regulation of the anti-tumour immune response by cancer-associated fibroblasts. Semin Cancer Biol. 2014;25:69-77.

184. Maxwell PJ, Neisen J, Messenger J, Waugh DJ. Tumor-derived CXCL8 signaling augments stroma-derived CCL2-promoted proliferation and CXCL12-mediated invasion of PTEN-deficient prostate cancer cells. Oncotarget. 2014;5(13):4895-4908.

185. Hwang TL, Lee LY, Wang CC, Liang Y, Huang SF, Wu CM. CCL7 and CCL21 overexpression in gastric cancer is associated with lymph node metastasis and poor prognosis. World J Gastroenterol. 2012;18(11):1249-1256.

186. Li T, et al. Hepatocellular carcinoma-associated fibroblasts trigger NK cell dysfunction via PGE2 and IDO. Cancer Lett. 2012;318(2):154-161.

187. Inada M, et al. Direct Melanoma Cell Contact Induces Stromal Cell Autocrine Prostaglandin E2-EP4 Receptor Signaling That Drives Tumor Growth, Angiogenesis, and Metastasis. J Biol Chem. 2015;290(50):29781-29793.

188. Mangoni M, et al. A PPAR-gamma agonist protects from radiation-induced intestinal toxicity. United European Gastroenterol J. 2017;5(2):218-226.

189. Mangoni M, et al. A PPAR gamma agonist protects against oral mucositis induced by irradiation in a murine model. Oral Oncol. 2017;64:52-58.

190. Deng W, Meng Z, Sun A, Yang Z. Pioglitazone suppresses inflammation and fibrosis in nonalcoholic fatty liver disease by down-regulating PDGF and TIMP-2: Evidence from in vitro study. Cancer Biomark. 2017;20(4):411-415.

191. Sherman $\mathrm{MH}$, et al. Vitamin D receptor-mediated stromal reprogramming suppresses pancreatitis and enhances pancreatic cancer therapy. Cell. 2014;159(1):80-93.

192. Dembinski JL, et al. Tumor stroma engraftment of gene-modified mesenchymal stem cells as anti-tumor therapy against ovarian cancer. Cytotherapy. 2013;15(1):20-32. 